\title{
Damage Assessment of Airport Target Based on Uncertain Information
}

\author{
Xia Chen and Xingchao Yu \\ School of Automation, Shenyang Aerospace University 110136, Shenyang, China; \\ xiachen1108@163.com,yuxingchao2010@163.com
}

\begin{abstract}
With the development of high technology, the complication of battle environment and the acceleration of the battle rhythm, target damage assessment plays a very important role in the modern war. Because the target damage information is uncertain information, it is a challenge on target damage assessment problem. To the problem of airport damage assessment, a new solution is proposed. The Fuzzy Comprehensive Evaluation method is given, and the two-level fuzzy comprehensive evaluation model based on uncertain information is proposed in this paper. The damage effect of airport target is evaluated by the two-level fuzzy comprehensive evaluation model. And the method of airport target damage grade is given by the compare of interval numbers. In the last, the effectiveness of this method is verified by the example.
\end{abstract}

Keywords: Fuzzy Comprehensive Evaluation; Analytic Hierarchy Process (AHP); Damage effect; Compare methods of interval numbers

\section{Introduction}

Target damage effect assessment refers to the comprehensive assessment of target damage effect after fire strikes on enemy targets. Target damage effect assessment is the target damage degree on the battlefield for timely and accurate estimation; it must be done before the attack and provide scientific basis for fire fighting planning. In modern war, airpower plays an important role on the outcome of the war. To seize the airpower, the airport targets tend to be one of the most priority targets in a variety of combat, so the research on airport damage assessment issue has been the focus in many researchers. By using imaging process and pattern recognition techniques, Zhao Hong gave a practical damage information processing method in 2002. A computer damage assessment system is built up to support the decision making for missile's striking plan [1]. Li Xinqi, being aimed at the target analysis problem in surface to surface missile fire program, then drew into the idea of target function vulnerable degree, moreover proposes the idea of damage effect index model building which are based on target function vulnerable degree [2]. By system simulation method, a model for evaluating the aircraft taking-off and landing ability was introduced by Guan Aijie. By simulate the damage situlation of all the line of the airfield, the taking-off-landing ability can be dynamicly calculated at any time of the combat [3]. Jiang Hao was to divide the airport index system through Analytic Hierarchy Process, and to realize evaluating on airport damage effect by using two-level fuzzy synthesis evaluation model [4]. A mathematic model of Battle Damage Assessment based on the recognized and battled airfield is proposed by Su Gaofeng. The technology of the image analyzing and image understanding is used sufficiently. an important judging index, the probability of the flying-off or put-down of the airplane, is offered in order to estimate the damage degree of the airfield accurately [5]. In 2008, using the method of Bayesian Network, the airport damage assessment method based on Bayesian Network is given by Ma Zhijun [6]. Based on the airport target features, considering interactions among physical damage effects propose a principle of selecting effects and construct a 
network-based evaluation model against airfield targets' characteristics, Li Dawei gave the evaluation method of airport damage effect based on network graph [7]. An airport damage assessment algorithm based visible images was discussed basically by Gao Pengfei. According to the basic conditions that airport runways must be satisfied in wartimes, a quantitative algorithm of airport runways damage assessment was putted forward [8]. Fan Yangtao, in 2009, according to the analysis of the airport target vulnerability, determine the damage indicator of each sub goal, using the theory of fuzzy mathematics, calculated the support capacity index of each sub target, established the operational effectiveness quantitative evaluation model of the airport damage [9]. By detecting technology and the principal component analysis method, to detect the change of remote sensing images before and after damage, the damage change information is extracted accurately and effectively, and combined with the damage effect evaluation model, Yan Jie put forward the overall operational performance evaluation method [10].

It is necessary to point out that, during the war, the collected information accepted by the radar, sensor etc. is often uncertain, there are lots of literature which evaluate the target damage grade by the method of fuzzy comprehensive evaluation, the fuzziness of the information are considered in the literature, but the uncertainty of information are still not considered. Aiming at the airport target damage evaluation problem about uncertain information, an analytic method is proposed in this paper. According to the method of fuzzy comprehensive evaluation to evaluate the airport target damage grade, a comprehensive evaluation method of the final target damage evaluation grade of uncertainty problem is given to ensure the accuracy of target damage grade evaluation.

\section{The Consists of Airport Target System}

From the plane taking off to completing the mission to return to the airport, it all depends on the airport facilities, if the airport facilities are attacked, the functions of taking off and landing will be damaged to varying degrees, air supremacy will be effected, eventually it may lead to operational failure. So the study of the effect of airport damage assessment is important for military application.

The airport is an important place for takeoff, landing, taxiing, park and organizational guarantee of aircraft activity, is an important basis of air combat and military training activities. There are many facilities in the airport, generally there are mainly four: flight area, aircraft parking facilities, communication and command facilities, and aircraft supply facilities. In the four facilities, flight area is the most basic facility.

Four facilities can be subdivided into some sub systems. (1) Flight area: the most important function of the part is able to land and take off for aircraft, the system is composed of the subsystems, such as runway ,taxiway ,contact way; (2) Aircraft parking facilities: storage and concealment is the main function of this part, it is composed by parking apron ,aircraft cave ,aircraft shelters; (3) Communication and command facilities: the important function of the part is allowing the airplane flight normal and land safely, it is composed by the flight control room, landing navigation equipment (beacon towers and directional table, landing radar, instrument landing system) subsystem, control tower and so on; (4) Aircraft supply facilities: the supply and maintenance of aircraft is the main function of the part, it is composed by oil depot, ammunition depot, material depot, aircraft repair factory and so on. System of airport target is given by the Table 1 [11]. 
Table 1. Airport Target System

\begin{tabular}{cc}
\hline Airport target & The two-level subsystem \\
\hline flight area $U_{1}$ & runway $u_{11}$, taxiway $u_{12}$, contact way $u_{13}$ \\
aircraft parking facilities $U_{2}$ & parking apron $u_{21}$, aircraft cave $u_{22}$, aircraft shelters $u_{23}$ \\
communication and & flight control room $u_{31}$, landing navigation equipment $u_{32}$, \\
command facilities $U_{3}$ & control tower $u_{33}$ \\
aircraft supply facilities $U_{4}$ & oil depot $u_{41}$, ammunition depot $u_{42}$, material $\operatorname{depot} u_{43}$, \\
& aircraft repair factory $u_{44}$ \\
\hline
\end{tabular}

\section{Evaluation Model of Airport Target Damage Effect}

Assuming that the damage evaluation set of airport target is $V=\left\{V_{1}, V_{2} \cdots, V_{n}\right\}, V_{i}$ is the damage grade of target, respectively, $V_{1}$ is no damage, $V_{2}$ is mild damage, $V_{3}$ is moderate damage, $V_{4}$ is serious damage, $V_{5}$ is target destroyed, the description of the damage grade is given by the Table $2[12,13]$.

Table 2. The Damage Grade Description

\begin{tabular}{|c|c|c|}
\hline \multicolumn{2}{|c|}{$\begin{array}{l}\text { The damage } \\
\text { grade }\end{array}$} & Language description \\
\hline$V_{1}$ & $\begin{array}{c}\text { No } \\
\text { damage }\end{array}$ & $\begin{array}{l}\text { Target intact, or minor damage, needn't to repair, the ratio of the } \\
\text { overall system performance decrease less than } 5 \% \text {. }\end{array}$ \\
\hline$V_{2}$ & $\begin{array}{r}\text { Mild } \\
\text { damage }\end{array}$ & $\begin{array}{l}\text { The damage is light, but not timely repairing will affect the } \\
\text { tactical performance of the system, need to repair or replace a small } \\
\text { amount of parts, and overall efficiency of the system to reduce the } \\
\text { rate at } 5 \% \sim 20 \% \text {. }\end{array}$ \\
\hline$V_{3}$ & $\begin{array}{l}\text { Moderat } \\
\text { e damage }\end{array}$ & $\begin{array}{l}\text { The damage is heavy, need to repair and replace more } \\
\text { components, and overall efficiency of the system to reduce the rate at } \\
20 \% \sim 50 \% \text {. }\end{array}$ \\
\hline$V_{4}$ & $\begin{array}{l}\text { Serious } \\
\text { damage }\end{array}$ & $\begin{array}{l}\text { The damage is serious, and the repair cycle is longer, more } \\
\text { consumption of equipment, and overall efficiency of the system to } \\
\text { reduce the rate at } 50 \% \sim 80 \% \text {. }\end{array}$ \\
\hline$V_{5}$ & $\begin{array}{r}\text { Target } \\
\text { destroyed }\end{array}$ & $\begin{array}{l}\text { Unable to repair or no repair of the value, and overall efficiency of } \\
\text { the system to reduce the rate above } 80 \%\end{array}$ \\
\hline
\end{tabular}

\subsection{Fuzzy Comprehensive Assessment of Airport Target}

Airport target is a complex system containing multiple sub goals, some sub goals damage can not be expressed by the exact information, has the very strong uncertainty and fuzziness, considering the hierarchical relationship between each sub goals, two-level fuzzy comprehensive assessment model to evaluate the airport damage effect are adopted in this paper.

\subsubsection{A Fuzzy Comprehensive Evaluation of Flight Area $U_{1}$}

Assuming that $u_{11}$ is the runway, $u_{12}$ is the taxiway, $u_{13}$ is the contact way, according to the interval information of two-level subsystem $U_{1}$ damage effect, so the damage information of flight area $U_{1}$ about two-level subsystem for comment set $V$ can be expressed as $R_{1}=\left(r_{i j}^{1}\right)_{i \times j}$, in the formula, $r_{i j}^{1}$ is the damage interval information of flight area $U_{1}$ about two-level subsystem $u_{1 i}$ for comment set $V_{j}$, it can be expressed as 
$r_{i j}^{1}=\left[r_{u_{i, j}}^{-}, r_{u_{i, j}}^{+}\right](i=1,2,3 ; j=1,2, \cdots, 5)$.

According to the important degree of the flight area $U_{1}$, it is assumed that the weight of two-level subsystem $U_{1}$ is $A_{U_{1}}=\left(a_{i}^{1}\right)_{1 \times i}, \quad a_{i}^{1}=\left[a_{u_{l_{i}}}^{-}, a_{u_{u_{i}}}^{+}\right](i=1,2,3)$.

You can get the one-level fuzzy comprehensive judgment of flight area $U_{1}$ :

$$
B_{1}=A_{u_{11}} \circ R_{1}=\left(b_{11}, b_{12}, \cdots, b_{15}\right)
$$

\subsubsection{The One-Level Fuzzy Comprehensive Judgment of Aircraft Parking Facilities $U_{2}$}

Assuming that $u_{21}$ is the parking apron, $u_{22}$ is the aircraft cave, $u_{23}$ is aircraft shelters, according to the interval information of damage effect of the two-level subsystem $U_{2}$,

so the damage information of aircraft parking facilities $U_{2}$ about two-level subsystem for comment set $V$ can be expressed as $R_{2}=\left(r_{i j}^{2}\right)_{i \times j}$, in the formula, $r_{i j}^{2}$ is the damage interval information of aircraft parking facilities $U_{2}$ about two-level subsystem $u_{2 i}$ for comment set $V_{j}$, it can be expressed as $r_{i j}^{2}=\left[r_{u_{2 i, j}}^{-}, r_{u_{2 i, j}}^{+}\right](i=1,2,3 ; j=1,2, \cdots, 5)$.

According to the function of the aircraft parking facilities $U_{2}$, it is assumed that the weight of two-level subsystem $U_{2}$ is $A_{U_{2}}=\left(a_{i}^{2}\right)_{1 \times i}, a_{i}^{2}=\left[a_{u_{2 i}}^{-}, a_{u_{2 i}}^{+}\right](i=1,2,3)$.

You can get the one-level fuzzy comprehensive judgment of aircraft parking facilities $U_{2}$ :

$$
B_{2}=A_{U_{2}} \circ R_{2}=\left(b_{21}, b_{22}, \cdots, b_{25}\right)
$$

\subsubsection{The One Level Fuzzy Comprehensive Judgment of Communication and Command Facilities $U_{3}$}

Assuming that $u_{31}$ is the flight control room, $u_{32}$ is the landing navigation equipment, $u_{33}$ is the control tower, according to the interval information of damage effect of the two-level subsystem $U_{3}$, so the damage information of communication and command facilities $U_{3}$ about two-level subsystem for comment set $V$ can be expressed as $R_{3}=\left(r_{i j}^{3}\right)_{i \times j}$, in the formula, $r_{i j}^{3}$ is the damage interval information of communication and command facilities $U_{3}$ about two-level subsystem $u_{3 i}$ for comment set $V_{j}$, it can be expressed as $r_{i j}^{3}=\left[r_{u_{i, j}}^{-}, r_{u_{3 i, j}}^{+}\right](i=1,2,3 ; j=1,2, \cdots, 5)$.

According to the important degree of the communication and command facilities $U_{3}$, it is assumed that the weight of two-level subsystem $U_{1}$ is $A_{U_{3}}=\left(a_{i}^{3}\right)_{1 \times i}$, $a_{i}^{3}=\left[a_{u_{3 i}}^{-}, a_{u_{3 i}}^{+}\right](i=1,2,3)$

You can get the one-level fuzzy comprehensive judgment of communication and command facilities $U_{3}$ :

$$
B_{3}=A_{U_{3}} \circ R_{3}=\left(b_{31}, b_{32}, \cdots, b_{35}\right)
$$

\subsubsection{The one Level Fuzzy Comprehensive Judgment of Aircraft Supply Facilities $U_{4}$}

Assuming that $u_{41}$ is the oil depot, $u_{42}$ is the ammunition depot, $u_{43}$ is the material depot, $u_{44}$ is the aircraft repair factory, according to the interval information of damage effect of the two-level subsystem $U_{4}$, so the damage information of aircraft supply 
facilities $U_{4}$ about two-level subsystem for comment set $V$ can be expressed as $R_{4}=\left(r_{i j}^{4}\right)_{i \times j}$, in the formula, $r_{i j}^{4}$ is the damage interval information of aircraft supply facilities $U_{4}$ about two-level subsystem $u_{4 i}$ for comment set $V_{j}$, it can be expressed as $r_{i j}^{4}=\left[r_{u_{i, j}}^{-}, r_{u_{i, j}}^{+}\right](i=1,2,3,4 ; j=1,2, \cdots, 5)$.

According to the important degree of the aircraft supply facilities $U_{4}$, it is assumed that the weight of two-level subsystem $U_{4}$ is $A_{U_{4}}=\left(a_{i}^{4}\right)_{1 \times i}, \quad a_{i}^{4}=\left[a_{u_{4 i}}^{-}, a_{u_{4 i}}^{+}\right](i=1,2,3,4)$

You can get the one-level fuzzy comprehensive judgment of aircraft supply facilities $U_{4}$ :

$$
B_{4}=A_{U 4} \circ R_{4}=\left(b_{41}, b_{42}, \cdots, b_{45}\right)
$$

\subsubsection{The Two-Level Fuzzy Comprehensive Assessment of the Airport Target}

Airport target $U$ contains a subsystem $U_{i}(i=1,2,3,4)$, the damage information $R=\left(B_{1}, B_{2}, B_{3}, B_{4}\right)^{T}$ of space target $U$ about two-level subsystem for comment set $V$ can be got by (1) (4). According to the important degree of the subsystem $U_{i}(i=1,2,3,4)$, it is assumed that the weight value of the subsystem $U_{i}(i=1,2,3,4)$ is $A=\left(A_{i}\right)_{1 \times i}$, where $A_{i}=\left[A_{i}^{-}, A_{i}^{+}\right](i=1,2,3,4)$ is the weight range of subsystem $_{U_{i}}$, we can get two-level evaluation vector of the damage grade of airport target, namely comprehensive evaluation vector

$$
B=A \circ R=\left(b_{1}, b_{2}, \cdots, b_{5}\right)
$$

\subsection{The Analysis of Airport Target Damage Evaluation}

According to (5) we can get the two-level evaluation vector $B$ of the airport damage grade, its each component is interval number, the interval numbers often contain each other, thus it is difficult to get the final damage level of target. According to the literature [14], the evaluation method of the final target damage effect is given by the comparison between interval numbers.

The situation of the two intervals is considered firstly, the damage range of the comment $V_{i}$ and $V_{j}$ of the two-level evaluation vector $B$ are $b_{i}=\left[b_{i}^{-}, b_{i}^{+}\right]$and $b_{j}=\left[b_{j}^{-}, b_{j}^{+}\right]$, we always assume that $b_{j}^{+} \geq b_{i}^{+}$. In particular, when $b_{i}^{-}=b_{i}^{+}$and $b_{j}^{-}=b_{j}^{-}$, then $b_{i}$ and $b_{j}$ are real. Therefore, $b_{i}$ and $b_{j}$ in the real axis location may have three kinds of cases, as shown in Figure 1, 2, 3.

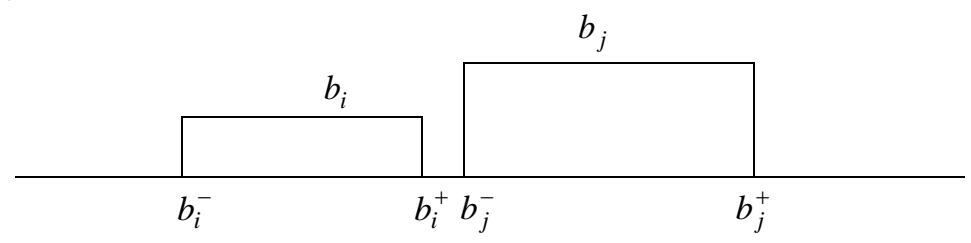

Figure 1. The Situation of $b_{i}^{+}<b_{j}^{-}$ 


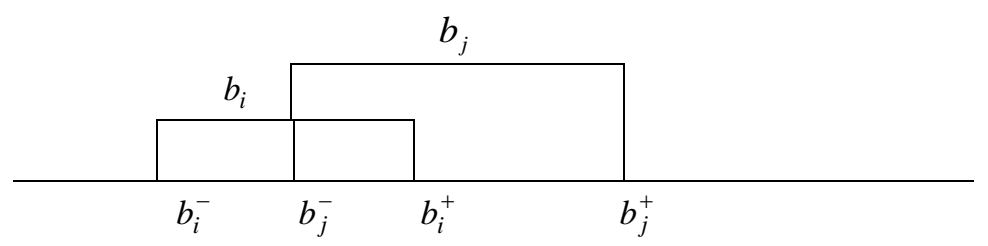

Figure 2. The Situation of $b_{i}^{-} \leq b_{j}^{-} \leq b_{i}^{+} \leq b_{j}^{+}$

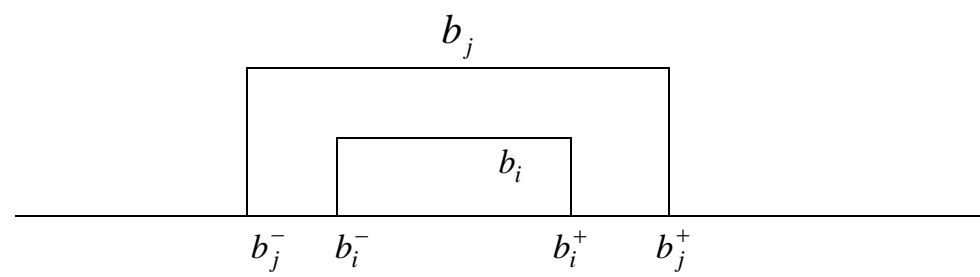

Figure 3. The Situation of $b_{j}^{-} \leq b_{i}^{-}<b_{i}^{+} \leq b_{j}^{+}$

(1) When $b_{i}$ and $b_{j}$ are real, the possibility degree of $b_{i}>b_{j}$ may be

$$
p\left(b_{i}>b_{j}\right)=\left\{\begin{array}{lc}
1 & b_{i}>b_{j} \\
1 / 2 & b_{i}=b_{j} \\
0 & b_{i}<b_{j}
\end{array}\right.
$$

(2)When $b_{i}$ and $b_{j}$ are intervals at the same time or one is an interval number, the possibility degree of $b_{i} \geq b_{j}$ may be

$$
p\left(b_{i} \geq b_{j}\right)=\max \left\{1-\max \left(\frac{b_{j}^{+}-b_{i}^{-}}{\left(b_{i}^{+}-b_{i}^{-}\right)+\left(b_{j}^{+}-b_{j}^{-}\right)}, 0\right), 0\right\}
$$

It is not difficult to see that, the following conclusions can be drawn by Figure 1, 2, 3 and the definition of possibility degree:

(1) The situation of Figure 1 , namely the situation of $b_{i}^{+}<b_{j}^{-}$, then $p_{b_{i}>b_{j}}=0$ and $p_{b_{j}>b_{i}}=1$. That $b_{j}$ is superior to $b_{i}$, the possibility degree is 1 ; Instead the possible degree of $b_{i}$ is superior to $b_{j}$ is 0 .

(2) The situation of Figure 2, namely the situation of $b_{i}^{-} \leq b_{j}^{-} \leq b_{i}^{+} \leq b_{j}^{+}$, then $p_{b_{i}>b_{j}} \leq 0.5$ and $p_{b_{j}>b_{i}} \geq 0.5$. That $b_{j}$ is superior to $b_{i}$, the possibility degree is equal or less than 0.5 ; the possible degree of $b_{i}$ is equal or greater than 0.5 .

(3) The situation of Figure 3, namely the situation of $b_{j}^{-} \leq b_{i}^{-}<b_{i}^{+} \leq b_{j}^{+}$, there are three possible cases:

(1) When $b_{i}^{-}-b_{j}^{-}=b_{j}^{+}-b_{i}^{+}$, that is to say $p_{b_{i}>b_{j}}=p_{b_{j}>b_{i}}=0.5$, namely the possible degree of $b_{i}$ is superior to $b_{j}$ and $b_{j}$ is better than $b_{i}$ may be equal;

(2) When $b_{i}^{-}-b_{j}^{-}>b_{j}^{+}-b_{i}^{+}$, that is to say $p_{b_{i} \succ b_{j}}>0.5, p_{b_{j}>b_{i}}<0.5$, that the possibility degree of $b_{i}$ is superior to $b_{j}$ is greater than 0.5 , the possibility degree of $b_{j}$ is superior to $b_{i}$ is less than 0.5 ;

(3) When $b_{i}^{-}-b_{j}^{-}<b_{j}^{+}-b_{i}^{+}$, that is to say $p_{b_{i}>b_{j}}<0.5, \quad p_{b_{j}>b_{i}}>0.5$, that the possibility 
degree of $b_{i}$ is superior to $b_{j}$ is less than 0.5 , the possibility degree of $b_{j}$ is superior to $b_{i}$ is greater than 0.5 .

(4) Special situation, When $b_{i}^{-}=b_{i}^{+}$and $b_{j}^{-}=b_{j}^{-}$, namely $b_{i}$ and $b_{j}$ are real, the damage grade of final target can be obtained by comparing the size of $b_{i}$ and $b_{j}$ directly.

The possibility matrix $P=\left(p_{i j}\right)_{n \times n}$ can be got by the above 4 kinds of situation, where $p_{i j}=p_{b_{i}>b_{j}}$.

The ranking formula given in literature 15 is

$$
w_{i}=\left(\sum_{j=1}^{n} p_{i j}+n / 2-1\right) / n(n-1) \quad i=1,2, \cdots, n
$$

Thus, the ranking vector $w=\left(w_{1}, w_{2}, \cdots, w_{n}\right)^{T}$ is got, $w$ is the ranking result of the interval component in two-level evaluation vector $B$ of the airport damage based on possible degree, thus the ranking results of target damage grade can be obtained.

\section{Example of the Application}

This paper selects the target system for airport target, the evaluation process as the following,

(1) To determine the fuzzy evaluation matrix of two-level sub systems

The evaluation set is $V_{i}(i=1,2, \cdots, 5)$, each of the 5 elements of evaluation set is fuzzy subset of interval $[0,1]$, in order to reduce the influence of artificial factors, the target evaluation function between damage effect and evaluation grade is established by comparison and practice inspection, expression is as follows:

$$
V_{i}(x)=\left\{\begin{array}{lc}
0 & x \leq \xi_{i 1} \\
\frac{x-\xi_{i}}{\xi_{i+1}-\xi_{i}} & \xi_{i}<x \leq \xi_{i+1} \\
1 & x>\xi_{i+1}
\end{array}\right.
$$

In the formula, $i=1,2, \cdots, 5, \xi_{1} \sim \xi_{6}$ are respectively $0.01,0.05,0.2,0.5,0.8,0.95$.

Based on the damage situation of airport target, 13 two-level subsystems were evaluated, the damage effect range as shown in Table 3:

\section{Table 3. The Damage Effect Interval of Subsystem}

\begin{tabular}{cc}
\hline Two-level subsystem & $\begin{array}{c}\text { The damage effect interval of two-level } \\
\text { subsystem }\end{array}$ \\
\hline runway $u_{11}$ & {$[0.85,0.98]$} \\
taxiway $u_{12}$ & {$[0.49,0.55]$} \\
contact way $u_{13}$ & {$[0.22,0.28]$} \\
parking apron $u_{21}$ & {$[0.71,0.83]$} \\
aircraft cave $u_{22}$ & {$[0.48,0.62]$} \\
aircraft shelters $u_{23}$ & {$[0.10,0.12]$} \\
flight control room $u_{31}$ & {$[0.65,0.65]$} \\
landing navigation equipment $u_{32}$ & {$[0.38,0.40]$} \\
control tower $u_{33}$ & {$[0.60,0.65]$} \\
oil depot $u_{41}$ & {$[0.38,0.40]$} \\
ammunition depot $u_{42}$ & {$[0.36,0.41]$} \\
material depot $u_{43}$ & {$[0.49,0.55]$} \\
aircraft repair factory $u_{44}$ & {$[0.79,0.88]$} \\
\hline
\end{tabular}


By the formula (9), one-level fuzzy evaluation matrix of the airdrome target is given, as the following:

$$
\begin{gathered}
R_{1}=\left(\begin{array}{lllll}
{[0.00,0.00]} & {[0.00,0.00]} & {[0.00,0.00]} & {[0.00,0.00]} & {[0.33,1.00]} \\
{[0.00,0.00]} & {[0.00,0.00]} & {[0.60,0.83]} & {[0.00,0.00]} & {[0.00,0.00]} \\
{[0.00,0.00]} & {[0.00,0.00]} & {[0.07,0.27]} & {[0.00,0.00]} & {[0.00,0.00]}
\end{array}\right) \\
R_{2}=\left(\begin{array}{lllll}
{[0.00,0.00]} & {[0.00,0.00]} & {[0.00,0.00]} & {[0.70,1.00]} & {[0.00,0.20]} \\
{[0.00,0.00]} & {[0.00,0.00]} & {[0.93,1.00]} & {[0.00,0.40]} & {[0.00,0.00]} \\
{[0.00,0.00]} & {[0.33,0.47]} & {[0.00,0.00]} & {[0.00,0.00]} & {[0.00,0.00]}
\end{array}\right) \\
R_{3}=\left(\begin{array}{lllll}
{[0.00,0.00]} & {[0.00,0.00]} & {[0.00,0.00]} & {[0.50,0.50]} & {[0.00,0.00]} \\
{[0.00,0.00]} & {[0.00,0.00]} & {[0.60,0.83]} & {[0.00,0.00]} & {[0.00,0.00]} \\
{[0.00,0.00]} & {[0.00,0.00]} & {[0.00,0.00]} & {[0.33,0.50]} & {[0.00,0.00]}
\end{array}\right) \\
R_{4}=\left(\begin{array}{lllll}
{[0.00,0.00]} & {[0.00,0.00]} & {[0.60,0.67]} & {[0.00,0.00]} & {[0.00,0.00]} \\
{[0.00,0.00]} & {[0.00,0.00]} & {[0.53,0.70]} & {[0.00,0.00]} & {[0.00,0.00]} \\
{[0.00,0.00]} & {[0.00,0.00]} & {[0.97,1.00]} & {[0.00,0.17]} & {[0.00,0.00]} \\
{[0.00,0.00]} & {[0.00,0.00]} & {[0.00,0.00]} & {[0.97,1.00]} & {[0.00,0.53]}
\end{array}\right)
\end{gathered}
$$

(2) The one-level fuzzy evaluation vector of two-level subsystem

Based on the different function and importance of the two-level subsystems, the weight vector of the two-level subsystems can be obtained:

$$
\begin{aligned}
& A_{1}=\left(a_{11}, a_{12}, a_{13}\right)=([0.35,0.37],[0.20,0.22],[0.44,0.45]) \\
& A_{2}=\left(a_{21}, a_{22}, a_{23}\right)=([0.41,0.42],[0.50,0.52],[0.08,0.10]) \\
& A_{3}=\left(a_{31}, a_{32}, a_{33}\right)=([0.22,0.23],[0.35,0.37],[0.42,0.44]) \\
& A_{4}=\left(a_{41}, a_{42}, a_{43}, a_{44}\right)=([0.17,0.18],[0.29,0.30],[0.26,0.27],[0.27,0.29])
\end{aligned}
$$

The subsystem $U_{i}$ of one-level fuzzy evaluation vector can be obtained by (1) (4), as the following:

$$
\begin{aligned}
& B_{1}=A_{1} \circ R_{1}=([0.00,0.00],[0.00,0.00],[0.15,0.30],[0.00,0.00],[0.12,0.37]) \\
& B_{2}=A_{2} \circ R_{2}=([0.00,0.00],[0.03,0.04],[0.47,0.52],[0.29,0.63],[0.00,0.02]) \\
& B_{3}=A_{3} \circ R_{3}=([0.00,0.00],[0.00,0.00],[0.21,0.31],[0.25,0.34],[0.00,0.00]) \\
& B_{4}=A_{4} \circ R_{4}=([0.00,0.00],[0.00,0.00],[0.51,0.60],[0.26,0.34],[0.00,0.15])
\end{aligned}
$$

(3) Airport target damage grade comprehensive evaluation vector

Two-level fuzzy evaluation matrix of airport target damage grade is the following:

$$
R=\left(\begin{array}{l}
B_{1} \\
B_{2} \\
B_{3} \\
B_{4}
\end{array}\right)=\left(\begin{array}{l}
{[0.00,0.00],[0.00,0.00],[0.15,0.30],[0.00,0.00],[0.12,0.37]} \\
{[0.00,0.00],[0.03,0.04],[0.47,0.52],[0.29,0.63],[0.00,0.02]} \\
{[0.00,0.00],[0.00,0.00],[0.21,0.31],[0.25,0.34],[0.00,0.00]} \\
{[0.00,0.00],[0.00,0.00],[0.51,0.60],[0.26,0.34],[0.00,0.15]}
\end{array}\right)
$$

Based on the different importance degree of the airport target, the weight vector of the airport target is obtained:

$$
A=\left(A_{1}, A_{2}, A_{3}, A_{4}\right)=([0.32,0.33],[0.27,0.29],[0.21,0.22],[0.19,0.20])
$$

We can get the comprehensive assessment vector of airport target damage grade by the formula (5):

$$
B=A \circ R=([0.00,0.00],[0.01,0.01],[0.20,0.44],[0.18,0.33],[0.04,0.20])
$$

(4) The comprehensive analysis of target damage grade evaluation

The possibility degree matrix $P$ is obtained by the formula (6) $\sim(7)$ and the comprehensive analysis of target damage evaluation, as follows, 


$$
P=\left(\begin{array}{lllll}
0.50 & 0.00 & 0.00 & 0.00 & 0.00 \\
1.00 & 0.50 & 0.00 & 0.00 & 0.00 \\
1.00 & 1.00 & 0.50 & 0.66 & 1.00 \\
1.00 & 1.00 & 0.34 & 0.50 & 0.94 \\
1.00 & 1.00 & 0.00 & 0.06 & 0.50
\end{array}\right)
$$

The ranking vector $w=(0.100,0.150,0.283,0.264,0.203)^{T}$ is obtained by the formula (8), the damage grade ranking of the target may be $V_{3}>V_{4}>V_{5}>V_{2}>V_{1}$, so the most possible damage grade of the targets is "Moderate damage $V_{3}$ ".

\section{Conclusion}

According to the damage effect assessment problem of airport target, the model of airport target damage effect assessment based on uncertain information is established by using the fuzzy comprehensive judgement method, and using interval number comparison method, the reasonable analysis method of target damage grade of uncertainty is presented. At the same time, the effectiveness of the method is verified with specific examples. Using the method can assess airport target damage effect for battlefield which changed quickly, it can greatly cut down the assessment time to ensure that our military occupy the airpower time and to give scientific basis for fire fighting planning.

\section{Acknowledgements}

The research work reported in this paper is supported by the National Nature Science Foundation of China (No. 61074159), Technology Innovation Foundation of Shenyang (No. 14042200 \& No. 14231129), and thanks to the researchers for the suggestions, research samples and experimental data.

\section{References}

[1] Z. Hong, B. Yiming and L. Fugen, "Information processing technology of missile damage airdrome target", Fire Control \& Command Control, vol. 27, (2002), pp. 50-53

[2] L. Xingqi, Y. Shirong, L. Xiaoqiang and Z. Guozhong, "The Idea and Model of Damag eEffect Index's Seleetion Based on Target Function Vulnerable Degree", Journal of Projectiles, Rockets, Missiles and Guidance, vol. 25, (2005), pp. 702-705

[3] G. Aijie, W. Yunji, L Zengliang and Q. Cheng-long, "Simulation and Evaluation of Aircraft Taking-off and Landing Capacity on Airfield Attacked", Fire Control \& Command Control, vol. 31, (2006), pp. $51-53$

[4] J. Hao, C. Haoguang and X. Xuehua, "Evaluation of Damage Effect for Airport Objects Based on Two-Level Fuzzy Synthesis Evaluation Method", Ordnance Industry Automation, vol. 26, (2007), pp. $1-2$

[5] S. Gaofeng, W. Baoshu and M. Qiguang, "Study on the evaluation of the effectiveness of airport runway", Fire Control \& Command Control, vol. 33, (2008), pp. 46-47, 52

[6] M. Zhijun, J. Xisheng and C. Li, "Battle Damage Assessment based on Bayesian Network", Acta Armamentarii, vol. 29, (2008), pp. 1509-1513

[7] L. Dawei and Z. Wenjie, "Damage Effect Evaluation for Airfield Target based on Network", Ordnance Industry Automation, vol. 28, (2009), pp. 47-50

[8] G. Pengfei, "Study Of Airport Runways Damage Assessment And Design Of Sumi-Physical Simulation Platform”, Shanghai Jiao Tong University, (2009).

[9] F. Yangtao, Y. Ping, S. Wenxue, Z. Xinqi and S. Yong-Fu, "Effectiveness evaluation of combat damage based on the airport", Journal of Sichuan ordnance, vol. 30, (2009), pp. 93-95

[10] Y. Jie, L. Jianpo and T. Guangwei, "The Damage Assessment based on Remote Sensing Image Change Detection", Radio Engineering, vol. 40, (2010), pp. 30-31, 41

[11] Z. Ren. "The Military Airport", Beijing, Chinese People's Liberation Army Publishing House, (1979).

[12] L. Xinqi, X. Aihong and L. Hongxia, "Calculation and Assessment on Damage Effect of System Target", Acta Armamentarii, vol. 29, (2008), pp. 57-62.

[13] R. Wei, W. Yizhi and Z. Lei, "Assessment Research on Target Damage of the Marine Campaign", Ship Electronic Engineering, vol. 28, (2008), pp. 59-62,100. 
[14] W. Jiang and H. Dengshi, “An Review on Ranking Methods of Interval Numbers”, Systems Engineering, vol. 22, (2004), pp. 1-4.

[15] X. Zeshui, "Algorithm for priority of fuzzy complementary judgement matrix", Journal of Systems Engineering, vol. 16, (2001), pp. 311-314.

[16] Z. Jijun, "Research on Method for Ranking Interval Numbers", Operations Research and Management Science, vol. 12, (2003), pp. 18-22.

\section{Authors}

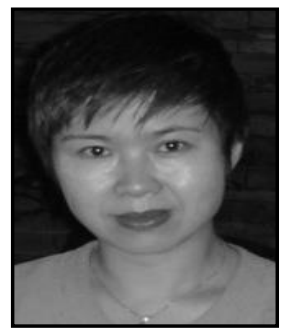

Xia Chen, she was born in 1962, she is a full professor in Shenyang Aerospace University. Her main research interests are measurement technique, automatic control, flight control, etc.

E-mail:xiachen1108@163.com

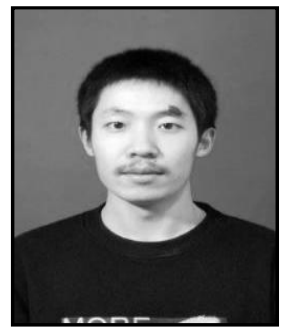

Xingchao Yu, he was born in 1989, he is a graduate student in Shenyang Aerospace University. His main research interest is the research of unmanned aerial vehicle (UAV) path planning technology.

E-mail:yuxingchao2010@163.com 